A) Check for updates

Cite this: Food Funct., 2020, 11 8939
Received 1st July 2020, Accepted 3rd September 2020

DOI: $10.1039 /$ dOfo01718c

rsc.li/food-function

\section{Lactiplantibacillus plantarum WJL administration during pregnancy and lactation improves lipid profile, insulin sensitivity and gut microbiota diversity in dyslipidemic dams and protects male offspring against cardiovascular dysfunction in later life}

\author{
Keyth Sulamitta de Lima Guimarães, ${ }^{a}$ Valdir de Andrade Braga, ${ }^{\text {b }}$ \\ Sylvana I. S. Rendeiro de Noronha, ${ }^{C}$ Whyara Karoline Almeida da Costa, ${ }^{d}$

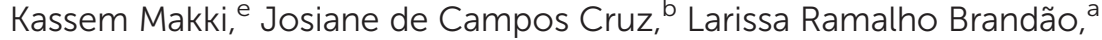 \\ Deoclecio Alves Chianca Junior, ${ }^{\mathrm{C}}$ Emmanuelle Meugnier, ${ }^{f}$ François Leulier, ${ }^{9}$ \\ Hubert Vidal, ${ }^{f}$ Marciane Magnani ${ }^{d}{ }^{d}$ and José Luiz de Brito Alves (D) *a
}

\begin{abstract}
Background and aim: Maternal dyslipidemia is recognized as a risk factor for the development of arterial hypertension $(\mathrm{AH})$ and cardiovascular dysfunction in offspring. Here we evaluated the effects of probiotic administration of a specific strain of Lactiplantibacillus plantarum (WJL) during pregnancy and lactation on gut microbiota and metabolic profile in dams fed with a high-fat and high-cholesterol (HFHC) diet and its longterm effects on the cardiovascular function in male rat offspring. Methods and results: Pregnant Wistar rats were allocated into three groups: dams fed a control diet $(C T L=5)$, dams fed a HFHC diet $(D L P=5)$ and dams fed a HFHC diet and receiving $L$. plantarum WJL during pregnancy and lactation (DLP-Lp ${ }^{W J L}$ ). L. plantarum WJL $\left(1 \times 10^{9} \mathrm{CFU}\right)$ or vehicle $(\mathrm{NaCl}, 0.9 \%)$ was administered daily by oral gavage for 6 weeks, covering the pregnancy and lactation periods. After weaning, male offspring received a standard diet up to 90 days of life. Biochemical measurements and gut microbiota were evaluated in dams. In male offspring, blood pressure (BP), heart rate (HR) and vascular reactivity were evaluated at 90 days of age. Dams fed with a HFHC diet during pregnancy and lactation had increased lipid profile and insulin resistance and showed dysbiotic gut microbiota. Administration of L. plantarum WJL to dams having maternal dyslipidemia improved gut microbiota composition, lipid profile and insulin resistance in them. Blood pressure was augmented and vascular reactivity was impaired with a higher contractile response and a lower response to endothelium-dependent vasorelaxation in DLP male offspring. In contrast, male offspring of DLP-LP ${ }^{W J L}$ dams had reduced blood pressure and recovered vascular function in later life. Conclusion: Administration of $L$. plantarum WJL during pregnancy and lactation in dams improved gut microbiota diversity, reduced maternal dyslipidemia and prevented cardiovascular dysfunction in male rat offspring.
\end{abstract}




\section{Introduction}

Experimental and clinical evidence has demonstrated that maternal dyslipidemia during pregnancy and/or lactation is a major risk factor for the development of cardio-metabolic disorders in the offspring later in life. ${ }^{1-3}$ Using a maternal dyslipidemia model induced by a high-fat and high-cholesterol (HFHC) diet consumption during pregnancy and/or lactation, it has been shown that male offspring developed arterial hypertension (AH) linked with sympathetic overactivity, impaired baroreflex, augmented peripheral chemoreceptor sensitivity and endothelial dysfunction in later life. ${ }^{3-6}$

Therapeutic strategies during pregnancy and/or lactation to alleviate maternal dyslipidemia and its deleterious effects on blood pressure and cardiovascular function in offspring are not completely efficient. Indeed, therapies based on lipid-lowering drugs have not been included in the obstetric routine and not all pregnant women respond effectively to nutritional therapy. $^{7}$

High-fat diet intake and hyperlipidemia during pregnancy and/or lactation alters the composition of maternal-fetal gut microbiota, which is associated with impaired gut barrier integrity, and leads to the developmental programming of arterial hypertension. ${ }^{8-11}$ These findings suggest that interventions targeting the gut microbiota during pregnancy and breastfeeding periods could be considered as an important intervention window to prevent and reduce the risk of maternal lipid disturbances, thus protecting the offspring against the development of cardiovascular disorders later in life.

Recently, studies have showed that bioactive food, plant components or probiotic-based therapy aiming at modulating the gut microbiota composition can improve glucose and lipid metabolism in humans and dams during gestation and/or

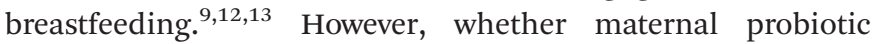
therapy is effective for the prevention of cardiovascular disorders in the offspring later in life remains to be elucidated.

Among probiotics, Lactobacillus strains are the most commonly used bacteria in studies looking at the impact of probiotic administration in the early life stages. $^{14}$ More recently, L. plantarum WJL, a bacterium originally isolated from the intestine of the fruit fly (Drosophila melanogaster), has been considered as a promising strain for the development of probiotic-based therapy as it showed beneficial impact on the health of juvenile rodents. ${ }^{15}$ These observations encouraged us to examine the effects of daily L. plantarum WJL administration during pregnancy and lactation on the gut microbiota and metabolic profile in dams fed with a HFHC diet, as well as, on the cardiovascular function, later in life, of male offspring.

\section{Materials and methods}

\section{Strain suspension preparation}

L. plantarum WJL was isolated from Drosophila and previously identified using whole genome sequence analysis. ${ }^{16,17}$ Stocks were stored at $-20{ }^{\circ} \mathrm{C}$ in Mann, Rogosa and Sharpe (MRS) broth (HiMedia, Mumbai, India) containing glycerol (SigmaAldrich, St Louis, USA; $20 \mathrm{~mL}$ per $100 \mathrm{~mL}$ ). The cell suspension was obtained from a culture grown overnight in MRS broth (HiMedia, Mumbai, India), and was anaerobically incubated at $37{ }^{\circ} \mathrm{C}$ (Anaerobic System Anaerogen, Oxoid Ltd, Wade Road, UK). Cells were harvested by centrifugation $(8000 g, 10 \mathrm{~min}$, $4{ }^{\circ} \mathrm{C}$ ), washed twice with a sterile saline solution and resuspended in the same diluent to obtain standard cell suspensions with optical density an (OD) reading at $660 \mathrm{~nm}\left(\mathrm{OD}_{660}\right)$ of 1.0. Fresh cell suspensions were prepared daily for experiments and corresponded to viable counts of approximately 9 $\log$ CFU mL ${ }^{-1}$.

\section{Ethical permissions and animals}

All animals used in the experiment received water and diet ad libitum and were maintained in polypropylene cages under a controlled temperature $\left(21 \pm 1^{\circ} \mathrm{C}\right)$, with humidity between $50-55 \%$ and a $12 \mathrm{~h}$ light-dark cycle. All experimental procedures were submitted and approved by the Institutional Animal Care and Use Committee of the Federal University of Paraíba (CEUA-UFPB protocol no. 4517240418) and followed the guidelines of the National Council for the Control of Animal Experimentation (CONCEA) and the International Principles for Biomedical Research Involving Animals.

\section{Experimental design}

Fifteen primiparous Wistar rats (Rattus norvegicus) at 90 days of age were mated 2:1 with fertile male rats. The presence of sperm in the vaginal smear was used to define the first day of pregnancy and then pregnant dams were transferred to individual cages and were divided in to three groups: group that received the control diet (CTL group, $n=5$ ), group that had HFHC diet-induced maternal dyslipidemia (DLP group, $n=5$ ) and DLP group that received $L$. plantarum WJL supplementation (DLP-Lp ${ }^{\mathrm{WJ} L}, n=5$ ). The control diet was prepared according to the American Institute of Nutrition - AIN-93G and the HFHC diet was purchased from Rhoster® (Araçoiaba da Serra, São Paulo, Brazil). The diets were offered to dams during the pregnancy and lactation periods (Table 1 ).

In the DLP-Lp ${ }^{\mathrm{WJ}}$ group, L. plantarum WJL suspension $\left(10^{9}\right.$ $\left.\log \mathrm{CFU} \mathrm{mL} \mathrm{mL}^{-1}\right)$ was administered by oral gavage $\left(1 \mathrm{~mL}^{\text {day }}{ }^{-1}\right)$ during the pregnancy and lactation periods. Saline solution (NaCl, 0.9\%) was administered as placebo to CTL and DLP dams during pregnancy and lactation periods. At $24 \mathrm{~h}$ after birth, the offspring were randomly adjusted to eight pups (4 males and 4 females) per litter. Up to $48 \mathrm{~h}$ after birth, L. plantarum WJL was not administered to dams to avoid maternal stress. At weaning, male offspring were housed (four per cage) and had free access to a commercial chow diet (NuviLab, São Paulo, Brazil) and water ad libitum.

The CTL, DLP and DLP-Lp ${ }^{\mathrm{WJ}}$ experimental groups were formed with one or two male offspring from each litter. The body weight of dams was monitored every week during pregnancy and lactation and every month for male offspring. The body weights and body lengths were used to determine the 
Table 1 Composition of control and dyslipidaemic diets offered to dams during pregnancy and lactation

\begin{tabular}{|c|c|c|}
\hline \multirow[b]{2}{*}{ Ingredients ( $\mathrm{g}$ per $100 \mathrm{~g}$ ) } & \multicolumn{2}{|l|}{ Diets } \\
\hline & Control (AIN-93G) ${ }^{a}$ & Dyslipidemic $^{b}$ \\
\hline Corn starch & 39.75 & 33.09 \\
\hline Dextrinized corn starch & 13.20 & 15.50 \\
\hline Casein $^{d}$ & 20.00 & 19.86 \\
\hline Sucrose & 10.00 & 6.00 \\
\hline Soybean oil & 7.00 & 3.00 \\
\hline Animal fat (lard) & - & 6.00 \\
\hline Non-hydrolyzed vegetable fat & - & 5.00 \\
\hline Sigma cholesterol & - & 1.00 \\
\hline Sigma colic acid & - & 0.50 \\
\hline Cellulose & 5.00 & 5.00 \\
\hline Mineral mix 93G & 3.50 & 3.50 \\
\hline Vitamin mix & 1.00 & 1.00 \\
\hline L-Cystine & 0.30 & 0.30 \\
\hline Choline bitartrate & 0.25 & 0.25 \\
\hline$t-\mathrm{BHQ}^{c}$ & 0.014 & 0.014 \\
\hline Calories $\left(\mathrm{kcal} \mathrm{g}^{-1}\right)$ & 3.96 & 4.34 \\
\hline Carbohydrates (kcal\%) & 63.62 & 50.32 \\
\hline Proteins (kcal\%) & 20.47 & 18.58 \\
\hline Lipids (kcal\%) & 15.92 & 31.11 \\
\hline
\end{tabular}

${ }^{a}$ Adapted from Reeves; Nielsen; Fahey (1993). ${ }^{b}$ Rhoster - Industry and Trade Ltd. ${ }^{c} t$-BHQ: tert-butylhydroquinone. ${ }^{d}$ Casein showed $85 \%$ purity ( $85 \mathrm{~g}$ protein for each $100 \mathrm{~g}$ casein).

body mass index [BMI, body weight $(\mathrm{g}) /$ length $\left.^{2}\left(\mathrm{~cm}^{2}\right)\right]$ anthropometrical parameter of the Lee index (cube root of body weight $(\mathrm{g}) /$ nose-to-anus length $(\mathrm{cm})$ ), according to a previous study. ${ }^{18}$

After pregnancy and lactation periods, biochemical measurements, glucose and insulin tolerance tests and gut microbiota composition were evaluated in dams. In male offspring, blood pressure (BP), heart rate (HR) and vascular reactivity were evaluated at 90 days of age.

\section{Glucose and insulin tolerance tests}

The oral glucose tolerance (OGTT) and insulin tolerance (ITT) tests were performed in rats fasted overnight. For OGTT, a glucose load ( $2 \mathrm{~g}$ per $\mathrm{kg}$ of body weight) was administered by oral gavage. Blood samples were taken from the tail vein before glucose administration and, subsequently, at 15, 30, 60, 90 and $120 \mathrm{~min}$. ITT was performed after $24 \mathrm{~h}$ of OGTT, following an intraperitoneal insulin injection ( 0.75 UI per $\mathrm{kg}$ body weight); blood glucose concentration was measured before (0 min) and after (30, 60, 90 and $120 \mathrm{~min})$ the insulin injection. Measurements of blood glucose concentration were performed using an Accu-Check glucometer (Bayer®).

\section{Biochemical measurements}

Blood samples were collected after an overnight fast. Blood was centrifuged at $300 \mathrm{~g}$ for $15 \mathrm{~min}$ at room temperature. Serum measurements for total cholesterol (TC), HDL-cholesterol, LDL-cholesterol and triglyceride concentrations were performed using appropriate enzymatic colorimetric kits according to the manufacturer's instructions (Bioclin, Belo Horizonte - Minas Gerais - Brazil).

\section{Maternal gut microbiota analysis}

At the end of lactation, fecal samples were collected from the colon after the sacrifice of dams and stored in a $-80^{\circ} \mathrm{C}$ freezer. Total DNA was extracted with a QIAmp PowerFecal ${ }^{\circledR}$ DNA Kit, and for each sample, a region of approximately 426 bp encompassing the V3 and V4 hypervariable regions of the 16S rDNA gene was targeted for sequencing. To amplify and sequence the V3-V4 hypervariable region of the 16S rDNA gene, the primers used were 16S-V3F [CCTACGGGNGGCWGCAG] and 16S-V4R [GGACTACHVGGGTWTCTAAT].

To prevent problems due to Illumina low-diversity libraries, each index sequence differed from the others by at least two nucleotides, and each nucleotide position in the sets of indices contained approximately $25 \%$ of each base (Table 2). For the preparation of the libraries, "Illumina TruseqDNA Sample Preparation v2" was used, labeling each sample with a bar code. Sequencing was performed using the equipment Illumina Miseq2000. More than 100000 reads per sample were generated, commonly recognized as sufficient for metagenomic research. The sequencing was performed using the Illumina MiSeq equipment purchased from the Biomnigene company (https://www.biomnigene.fr/en/).

\section{Taxonomic assignment obtained by 16S rRNA gene sequencing analysis}

Quality checks of the initial sequences and quality filtering were performed using the FastQC and Trimmomatic (0.36) tools, respectively. The bioinformatics analysis was performed using the Quantitative Insights Into Microbial Ecology (QIIME2 version 2019.4) platform. Sequences were first trimmed, denoised and chimera filtered using the DADA2 plugin with the following parameters (the non-specified parameters were used with the default values): trunc_len_f: 0; trunc_len_r: 0; trim_left_f: 10; trim_left_r: 10; n_reads_learn: 200000 .

The taxonomic classification was then achieved using the fit-classifier-naive-Bayes module with the default parameters and the Greengenes database (gg_13_8_otus.tar.gz classifier for $16 \mathrm{~S}$ rRNA). Native pipelines from QIIME 2 were used to perform alpha and beta analyses, and taxonomy analysis, and to generate Bray-Curtis PCoA biplots. ANCOM was used to assess the relative abundance inference over treatment. ${ }^{19,20}$

\section{Fecal L. plantarum quantification}

The presence of $L$. plantarum strains in the feces was assessed by qPCR using a Rotor-Gene Real-Time PCR system (Qiagen, Courtaboeuf, France) and the following primer sequences: LpF: 5'-ACGTTAGGGCTACTCGGCCA-3' and Lp-R: 5'-GCCTTCGCCGACCCCAATTA-3'. The qPCR cycling conditions consist of an initial heat activation at $95{ }^{\circ} \mathrm{C}$ for $30 \mathrm{~s}$, followed by 40 cycles of denaturation at $95{ }^{\circ} \mathrm{C}$ for $5 \mathrm{~s}$, and annealing and extension at $60{ }^{\circ} \mathrm{C}$ for $30 \mathrm{~s}$. Melting curve analysis was performed at the 


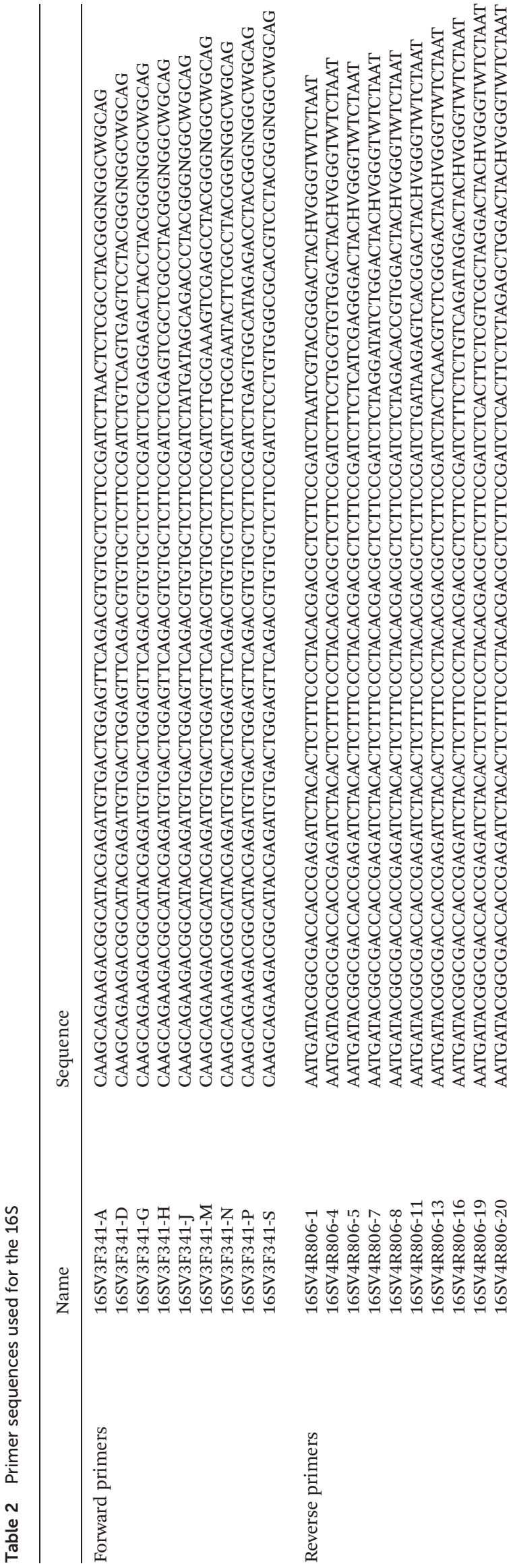

end of the amplification cycle to confirm the specificity of the amplification.

\section{Surgical procedure and blood pressure record}

At 90 days of age, male offspring rats ( $n=5-6$ per group) were anesthetized with ketamine $\left(80 \mathrm{mg} \mathrm{kg}{ }^{-1}\right.$, i.p.) and xylazine $\left(10 \mathrm{mg} \mathrm{kg}{ }^{-1}\right.$, i.p.) for the insertion of polyethylene catheters into the femoral artery and vein. The catheters were tunneled through the back of the neck and ketoprofen $\left(5 \mathrm{mg} \mathrm{kg}^{-1}\right)$ was injected subcutaneously. Rats underwent a period of surgical recovery for $24 \mathrm{~h}$. Arterial pressure (AP) and heart rate (HR) were recorded in conscious animals connected with the arterial catheter to a pressure transducer (LabChartTM Pro, ADInstruments, Bella Vista, NSW, Australia).

\section{Cardiovascular parameters}

The pulsatile arterial pressure (PAP) and HR were recorded for 50-60 min under baseline conditions. Values for systolic arterial pressure (SAP), diastolic arterial pressure (DAP) and mean arterial pressure (MAP) were calculated offline by selection of 10 min intervals for each animal (LabChartTM Pro, ADInstruments, Bella Vista, NSW, Australia).

\section{Endothelial function}

Mesenteric arteries from male offspring $(n=8)$ not subjected to surgical procedure for blood pressure recordings were isolated and mounted in myograph chambers (Model 620M, Danish Myo Technology, Aarhus, Denmark) for evaluating vasoconstriction and endothelium-dependent vasorelaxation as previously described. ${ }^{21}$

\section{Statistical analyses}

Results were expressed as mean \pm standard errors. KolmogorovSmirnov test was used to assess the distribution of the data. Oneway ANOVA, followed by Tukey's post-test and Kruskal-Wallis test, followed by Dunn's post-test were used according to the distribution of the data. For glucose and insulin tolerance tests and the anthropometrical variables, two-way ANOVA and Bonferroni's post hoc tests were applied. Statistical analyses were performed using the computational software Prism 5 (GraphPad Software, San Diego, CA). The difference was considered statistically significant when $p<0.05$.

\section{Results}

\section{Effects of $L$. plantarum WJL supplementation during pregnancy and lactation on body weight and metabolic profile in dams fed with a HFHC diet}

The body weight during pregnancy and lactation was similar among the groups (Table 3). As expected, dams fed with a HFHC diet during pregnancy and lactation showed significantly increased serum levels of cholesterol and LDL-cholesterol and reduced HDL-cholesterol (Table 3). Additionally, DLP dams developed intolerance to glucose and insulin resistance, as reflected by the increased area under the curve (AUC) 
Table 3 Assessment of body weight and biochemical variables in dams fed a control diet (CTL), high-fat and high-cholesterol diet induced dyslipidaemia (DLP), and DLP receiving $L$. plantarum WJL (DLP-Lp ${ }^{\text {WJL) }}$ during pregnancy and lactation

\begin{tabular}{llll}
\hline Variables & CTL & DLP & DLP-Lp \\
\hline Body weight (g) & & & \\
Pre-gestation & $203 \pm 2$ & $203 \pm 5$ & $200 \pm 3$ \\
Week 1 of gestation & $230 \pm 4$ & $224 \pm 7$ & $224 \pm 3$ \\
Week 2 of gestation & $260 \pm 6$ & $250 \pm 6$ & $240 \pm 6$ \\
Week 3 of gestation & $305 \pm 7$ & $290 \pm 8$ & $270 \pm 8$ \\
Week 1 of postpartum & $260 \pm 6$ & $230 \pm 8$ & $230 \pm 5$ \\
Week 2 of postpartum & $270 \pm 8$ & $245 \pm 6$ & $240 \pm 5$ \\
Week 3 of postpartum & $270 \pm 7$ & $250 \pm 4$ & $252 \pm 8$ \\
Biochemical variables & & & \\
Triglycerides (mg dL d $\left.^{-1}\right)$ & $115 \pm 17$ & $203 \pm 53$ & $89 \pm 9^{\#}$ \\
Total cholesterol (mg dL ${ }^{-1}$ ) & $85 \pm 4$ & $270 \pm 46^{*}$ & $154 \pm 12^{\# *}$ \\
LDL-cholesterol (mg dL $\left.{ }^{-1}\right)$ & $25 \pm 4$ & $230 \pm 66^{*}$ & $78 \pm 15^{\#}$ \\
HDL-cholesterol (mg dL ${ }^{-1}$ ) & $65 \pm 8$ & $42 \pm 8^{*}$ & $70 \pm 7^{\#}$ \\
OGTT (AUC) & $9049 \pm 399$ & $12455 \pm 721^{*}$ & $10163 \pm 383^{\#}$ \\
ITT (AUC) & $2827 \pm 174$ & $4087 \pm 470^{*}$ & $2247 \pm 116^{\#}$
\end{tabular}

${ }^{*} p<0.05$ indicates the difference in the mean values of the variable between the DLP or DLP-Lp ${ }^{\mathrm{WJL}}$ and CTL groups. ${ }^{*} p<0.05$ indicates the difference in the mean values of the variable between the DLP-Lp ${ }^{\mathrm{WJL}}$ and CTL groups. Abbreviations: LDL: low-density lipoprotein; HDL: high-density lipoprotein; OGTT: oral glucose tolerance test; ITT: insulin tolerance test; AUC: area under the curve.

during OGTT and ITT when compared with CTL dams (Table 3). Interestingly, daily administration of L. plantarum WJL during pregnancy and lactation significantly reduced serum triglycerides, cholesterol and LDL-cholesterol levels, while HDL-cholesterol levels were increased by the treatment (Table 3). These results were associated with better metabolic status of the animals as presented by a better clearance of blood glucose during OGTT and ITT (AUC) in DLP dams receiving L. plantarum WJL (Table 3 ).
Effects of $L$. plantarum WJL on gut microbiota in dams fed with a HFHC diet during pregnancy and lactation

To assess the impact of $L$. plantarum WJL supplementation on gut microbiota composition, we performed a 16S rRNA gene sequencing analysis and observed that HFHC diet significantly reduced the overall alpha diversity and altered the beta diversity of the gut bacterial community (Fig. 1a-d). Indeed, pairwise comparison based on the observed OTU index (Fig. 1a), a measure of richness, considering the number of species (or OTUs) revealed that DLP dams ( $p=$ $0.021)$ and DLP-Lp ${ }^{\mathrm{WJL}}$ dams $(p=0.020)$ had lower diversity compared to the CTL group. Similar finding was obtained using the Shannon index (Fig. 1b), which correspond to a measure of richness and evenness ecology. Beta diversity analysis or the Bray-Curtis dissimilarity matrix PCoA showed that the community structure was significantly different (PERMANOVA; pseudo $=2.582 ; p<0.005$ ) between the DLP and CTL groups (Fig. 1c).

Interestingly, L. plantarum WJL supplementation significantly improved the alpha diversity by the OTU index ( $p=$ 0.043) (Fig. 1a) and tended to improve the Shannon index without reaching statistical significance $(p=0.083)$. However, the bacterial supplementation failed to restore the beta diversity (Fig. 1c and d) and the pairwise comparison test showed that the CTL group was significantly different from the dams receiving $L$. plantarum WJL treatment ( $p=0.029$, Fig. 1d). Relative frequency analysis of microbes at the phyla level showed that Firmicutes and Fusobacteria were increased in the DLP group compared to the CTL group $(p<0.05$, Fig. 1e and f), while Bacteroidetes, Verrucomicrobia and Actinobacteria were reduced by the HFHC diet $(p<0.05$, Fig. 1f). Surprisingly, L. plantarum WJL supplementation did
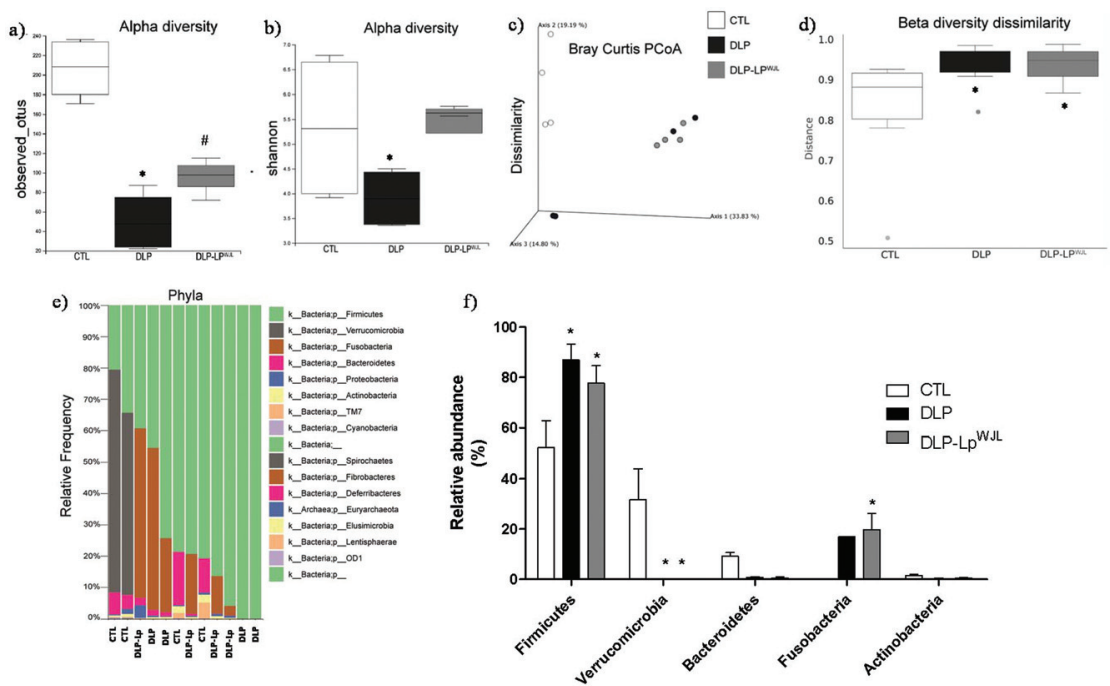

Fig. 1 Assessment of alpha diversity measurements in terms of the observed species (a), Shannon index (b), Bray-Curtis dissimilarity of relative abundance (c), beta diversity dissimilarity (d) and the relative frequency of the phylum (e and f) in dams fed with a control diet (CTL, $n=4)$, HFHC $\operatorname{diet}(\mathrm{DLP}, n=4)$, and DLP receiving $L$. plantarum WJL (DLP-Lp $\left.{ }^{\mathrm{WJ}}, n=4\right)$ during pregnancy and lactation. ${ }^{*} p<0.05$ indicates the difference in the mean values of the variable between the DLP or DLP-Lp ${ }^{\text {JJL }}$ and CTL groups. ${ }^{*} p<0.05$ indicates the difference in the mean values of the variable between the DLP-Lp ${ }^{W J L}$ and DLP groups. 


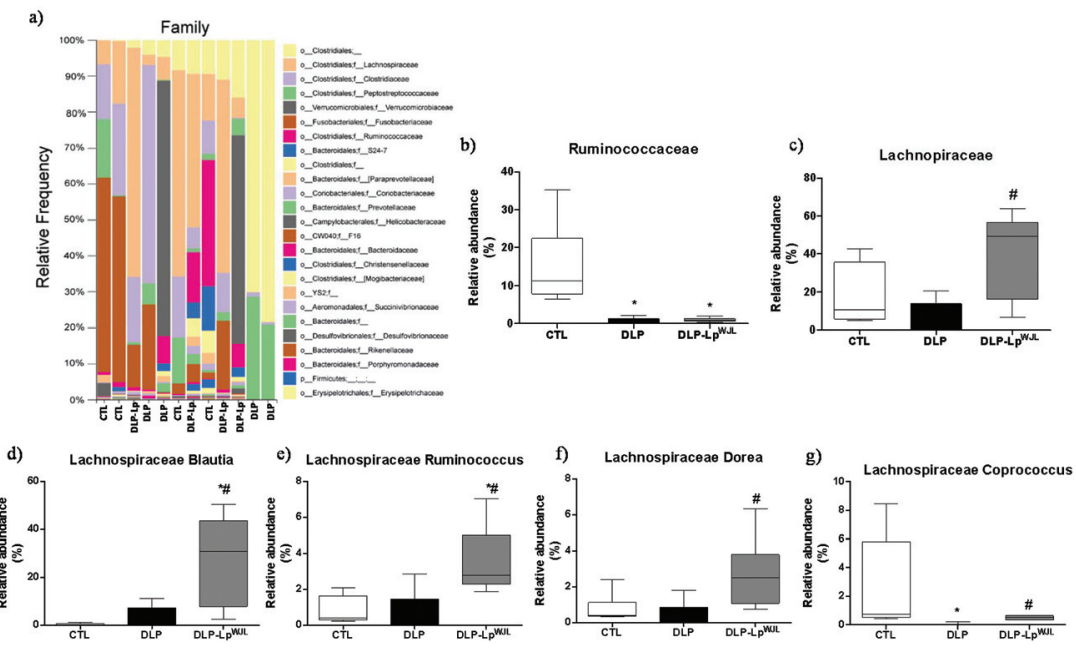

Fig. 2 Assessment of the relative frequency of the family (a), abundance of Rumincoccaceae (b) and Lachnospiraceae (c) families and Lachnospiraceae genera $(\mathrm{d}-\mathrm{g})$ in dams fed with a control diet $(C T L, n=4)$, HFHC diet (DLP, $n=4)$, and DLP receiving L. plantarum WJL (DLP-Lp WJL, $n=4$ ) during pregnancy and lactation. ${ }^{*} p<0.05$ indicates the difference in the mean values of the variable between the DLP or DLP-Lp ${ }^{\mathrm{WJL}}$ and CTL groups. ${ }^{\#} p<0.05$ indicates the difference in the mean values of the variable between the DLP-Lp ${ }^{\mathrm{WJL}}$ and DLP groups.

not restore the phyla relative abundance to the levels observed in the control group (Fig. 1e and f).

Analysis of microbes at the family level showed that L. plantarum WJL administration in DLP dams significantly augmented Lachnospiraceae, a family of short-chain fatty acid producers (Fig. 2a and c), which was accompanied by an increase in their genera abundance including Ruminococcus, Blautia, Dorea and Coprococcus (Fig. 2b-g).

In order to rule out the hypothesis that the modest effects on gut microbiota composition in the DLP-Lp ${ }^{\mathrm{WJL}}$ group are due to an unsuccessful bacterial supplementation, a quantification of the abundance of $L$. plantarum population was performed by qPCR in fecal samples of the experimental groups. The analysis showed significantly increased levels of L. plantarum population in the feces of the DLP-Lp ${ }^{\mathrm{WJL}}$ group compared to the DLP and CTL groups (Fig. 3), thus, reflecting a successful bacterial supplementation.

Effects of $\boldsymbol{L}$. plantarum WJL supplementation during maternal dyslipidemia on body weight and somatic growth in male rat offspring

Body weight of rat offspring in the DLP-Lp ${ }^{\mathrm{WJL}}$ group was decreased at 30, 60 and 90 days of age when compared to the CTL and DLP groups (Fig. 4a). In addition, body length of the DLP-Lp ${ }^{\text {WJL }}$ offspring was reduced at 30 and 90 days of age when compared to the CTL and DLP groups (Fig. 4b). Despite alterations in body weight and body length, the BMI and Lee index were similar among the groups at 90 days of age (Fig. $4 \mathrm{c}$ and d).

Effects of $L$. plantarum WJL during maternal dyslipidemia on blood pressure and heart rate in male rat offspring

The SAP, DAP and MAP were increased in the DLP male offspring when compared to the respective CTL groups $(p<$ 0.05 , Fig. 5a-c). Male offspring of DLP dams that received

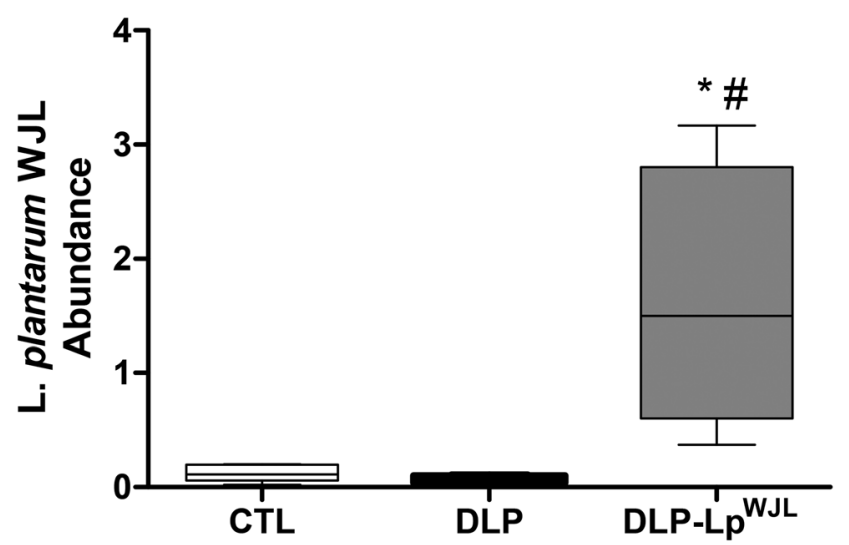

Fig. 3 Quantification of L. plantarum population by qPCR in the faecal samples of dams. Control (CTL, $n=4)$, HFHC diet (DLP, $n=4)$ and DLP receiving $L$. plantarum during pregnancy and lactation (DLP-Lp ${ }^{\text {WJL }}$, $n=4)$. Data are shown as mean \pm SEM, analyzed by one-way ANOVA, and followed by Tukey's post-test. ${ }^{\star} p<0.05$ indicates the difference in the mean values of the variable between the DLP-Lp ${ }^{\text {WJL }}$ and CTL groups. ${ }^{*} p<0.05$ indicates the difference in the mean values of the variable between the DLP-Lp ${ }^{\text {WJL }}$ and DLP groups.

L. plantarum WJL during pregnancy and lactation had reduced SAP and MAP when compared to the offspring of DLP dams without treatment $(p<0.05$, Fig. 5a-c). No differences were observed in HR among the groups (Fig. 5d).

Effects of $L$. plantarum WJL during maternal dyslipidemia on vascular reactivity in male rat offspring

The vascular responses assessed in mesenteric artery rings with functional endothelium using phenylephrine are shown in Fig. 6a. The potency indicated by pD2 was increased in the DLP and DLP-Lp ${ }^{\mathrm{WJL}}$ groups when compared with the CTL 
a)

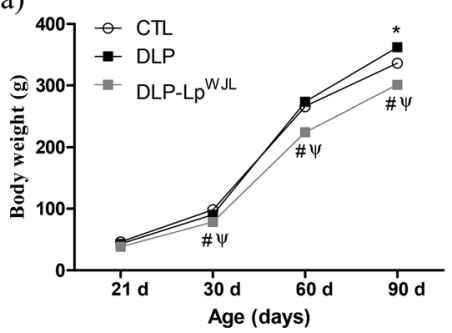

c)

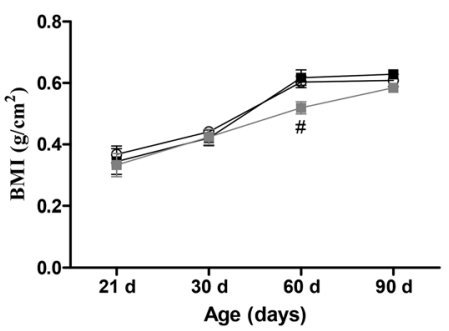

b)

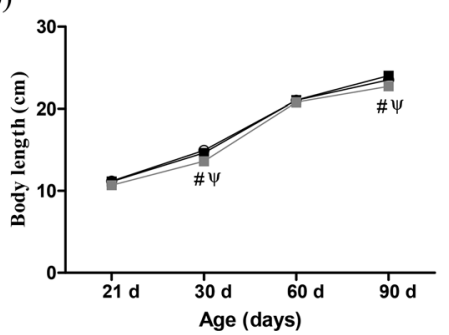

d)

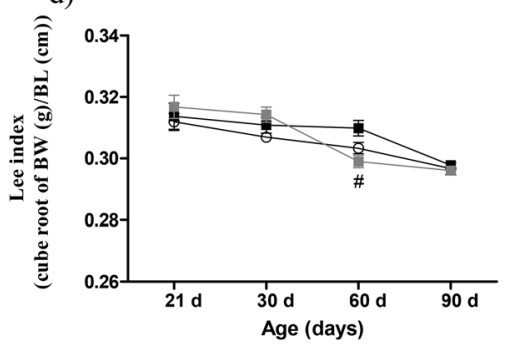

Fig. 4 Effects of $L$. plantarum WJL administration during maternal dyslipidaemia on the anthropometrical variables in male rat offspring. Assessment of body weight (a), body length (b), body mass index (BMI, c) and Lee index (d) in male offspring of dams fed a control diet (CTL), the high-fat and high-cholesterol diet induced dyslipidaemia (DLP) group, and the DLP group receiving L. plantarum WJL (DLP-Lp ${ }^{\text {WJL) }}$ during pregnancy and lactation. ${ }^{*} p<0.05$ indicates the difference in the mean values of the variable between the DLP and CTL groups; ${ }^{\#} p<0.05$ indicates the difference in the mean values of the variable between the DLP-LP ${ }^{\text {WJL }}$ and DLP groups; $\Psi$ indicates the difference in the mean values of the variable between the DLP-Lp ${ }^{\text {WJ }}$ and CTL groups.

a

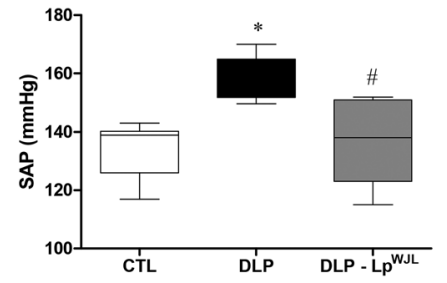

C

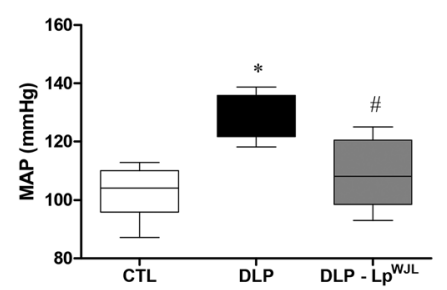

b

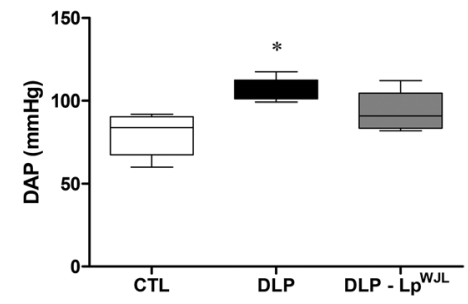

$\mathrm{d}$

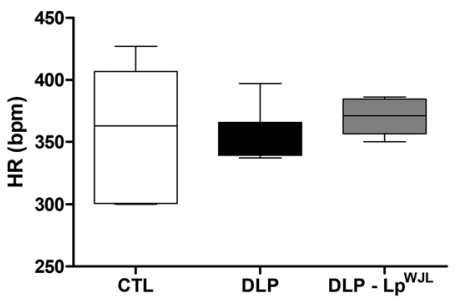

Fig. 5 Effects of $L$. plantarum WJL administration during maternal dyslipidaemia on blood pressure and heart rate in male rat offspring. Evaluations of resting systolic blood pressure (SAP, a), diastolic blood pressure (DAP, b), mean arterial pressure (MAP, c), and heart rate (HR, d) in male offspring at 90 days of age. Groups: control group (CTL, $n=6$ ), dyslipidaemic group (DLP, $n=6$ ) and DLP group of dams that received $L$. plantarum WJL during pregnancy and lactation (DLP-Lp ${ }^{\mathrm{WJL}}, n=5$ ). Data are shown as mean \pm SEM, analyzed by one-way ANOVA, followed by Tukey's post-test or Kruskal-Wallis and Dunn's post-test. ${ }^{*} p<0.05$ indicates the difference in the mean values of the variable between the DLP and CTL groups. ${ }^{\#} p<0.05$ indicates the difference in the mean values of the variable between the DLP-Lp ${ }^{W J L}$ and DLP groups.

offspring (Table 4). However, the DLP-Lp ${ }^{\mathrm{WJL}}$ group had reduced maximum effect $\left(E_{\max }\right)$ of phenylephrine in comparison with the DLP group (Table 4). The vascular relaxations assessed with functional endothelium using acetylcholine are shown in Fig. 6b. The potency indicated by pD2 was reduced in the DLP and DLP-Lp ${ }^{\mathrm{WJ}}$ groups when compared with the
CTL offspring (Table 4). The $E_{\max }$ of acetylcholine was reduced in the DLP group when compared to the CTL group, while the DLP-LP ${ }^{\mathrm{WJL}}$ group had recovered $E_{\max }$ in comparison with the DLP offspring (Table 4).

The vascular relaxations assessed in the rings without functional endothelium using sodium nitroprusside are shown in 
a)

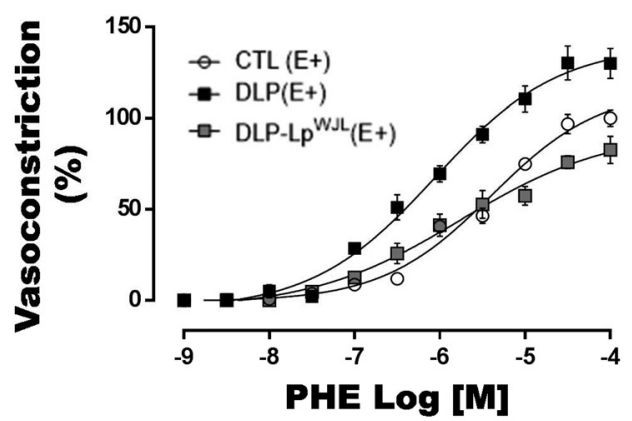

b)
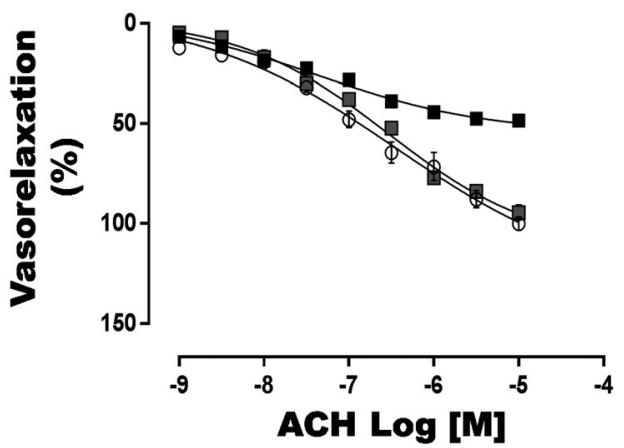

c)

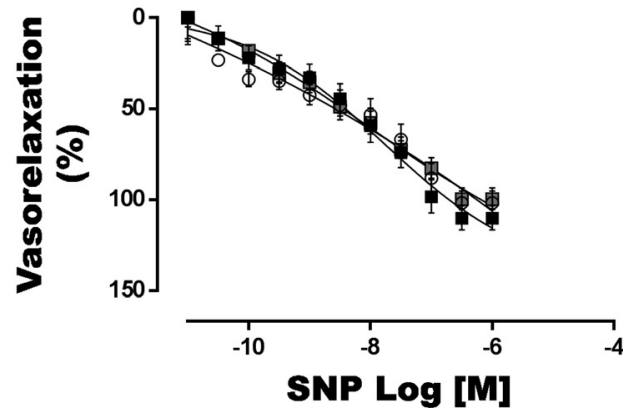

Fig. 6 Effects of L. plantarum WJL administration during maternal dyslipidemia on endothelial function in male rat offspring. Evaluation of vascular reactivity in the mesenteric artery rings. Dose-response curve for phenylephrine $(\mathrm{PHE}, \mathrm{a})$, the concentration-response curve for acetylcholine $(\mathrm{ACH}, \mathrm{b})$ in rings with functional endothelium, and the dose-response curve for sodium nitroprusside (SNP, c) in rings without functional endothelium Data are presented as mean \pm SEM. Groups: control group (CTL, $n=8$ ), dyslipidemic group (DLP, $n=8$ ) and DLP offspring from dams that received $L$. plantarum WJL during pregnancy and lactation (DLP-Lp ${ }^{\text {WJL }}, n=8$ ).

Fig. 6c. The potency, indicated by pD2, and $E_{\max }$ were similar among the groups (Table 4).

\section{Discussion}

In the present study, we described that administration of L. plantarum WJL in dyslipidaemic dams during pregnancy and lactation modestly increased gut microbiota diversity, reduced dyslipidemia and improved glucose tolerance and insulin sensitivity in dams. Furthermore, maternal
L. plantarum WJL intervention protected male offspring against the development of arterial hypertension and cardiovascular dysfunction later in life.

Maternal dyslipidemia is a complex clinical condition associated with maternal-fetal complications and deleterious effects on offspring in later life. For example, supraphysiological levels of cholesterol during pregnancy and/or lactation has been associated with preeclampsia, ${ }^{22}$ increased risk for preterm birth ${ }^{23}$ and cardiovascular outcomes in offspring, such as greater risk of aortic and coronary atherosclerotic plaques, $^{24}$ endothelial dysfunction ${ }^{5,25}$ and arterial hypertension. ${ }^{3,4}$

Considering the impact of maternal dyslipidaemia on the development of cardiometabolic diseases in mother and offspring added to the extra limitation for the use of lipid-lowering medications during pregnancy, the development of safe therapeutic strategies capable of reducing supraphysiological dyslipidaemia during pregnancy and/or lactation might represent a window of opportunity to prevent cardiovascular disease development in offspring.

The therapeutic modulation of gut microbiota by the administration of probiotics during pregnancy neither increases nor decreases the risk of preterm birth or other infant and maternal adverse pregnancy outcomes ${ }^{26}$ indicating that probiotic prescription might be a safe therapy to recommend for pregnant women. Earlier studies suggested that probiotic administration may improve lipid profile by affecting their absorption or metabolism. Here, we showed that administration of $L$. plantarum WJL during pregnancy and lactation reduced the serum levels of cholesterol and LDL-cholesterol, increased HDL-cholesterol and reduced insulin resistance in dams fed with a HFHC diet. Although the underlying mechanism by which $L$. plantarum WJL reduced lipid profile and insulin resistance was not assessed in the present study, it has been proposed that Lactobacillus strains may exert some hypocholesterolemic effect through binding to cholesterol in intestinal lumen and increasing its excretion in feces. ${ }^{27}$ Other studies suggested that Lactobacillus strains may convert cholesterol to coprostanol through the action of bacterial cholesterol reductase, ${ }^{28}$ and potentially may reduce hepatic cholesterol synthesis. Alternatively, some of these bacteria possess a bile acid hydrolase activity and may interfere with cholesterol metabolism by impacting bile acid composition and production. ${ }^{29}$

It has been also proposed that potentially probiotics such as Lactobacillus may exert beneficial effects through the modulation of gut microbiota composition or function. ${ }^{9,30}$ Although based on a small sample size, our analysis of fecal bacterial community indicated that administration of L. plantarum WJL during pregnancy and lactation modestly improved bacterial richness and diversity in the microbial ecosystem of dams fed a HFHC diet. These results are in agreement with other studies, ${ }^{31,32}$ suggesting a positive effect conferred by L. plantarum on gut microbiota.

The consumption of HFHC diet during pregnancy and lactation altered the microbial communities at the phyla level 
Table 4 Assessment of vascular reactivity in the male offspring of dams fed a control diet (CTL), high-fat and high-cholesterol diet induced dyslipidaemia (DLP), and DLP receiving L. plantarum WJL (DLP-LpWJL) during pregnancy and lactation

\begin{tabular}{|c|c|c|c|c|c|c|}
\hline Groups & \multicolumn{2}{|c|}{ Contraction to PHE $(E+)$} & \multicolumn{2}{|c|}{ Relaxation to $\mathrm{ACH}(E+)$} & \multicolumn{2}{|c|}{ Relaxation to SNP $(E-)$} \\
\hline CTL & $100.0 \pm 4.51$ & $5.39 \pm 0.1$ & $100.0 \pm 3.1$ & $5.3 \pm 0.3$ & $101.5 \pm 6.3$ & $11.3 \pm 0.7$ \\
\hline DLP-Lp ${ }^{W J L}$ & $82.7 \pm 7.43^{* \#}$ & $5.75 \pm 0.2^{* \#}$ & $94.53 \pm 3.74^{\#}$ & $2.4 \pm 0.1^{*}$ & $99.53 \pm 5.9$ & $7.9 \pm 0.1$ \\
\hline
\end{tabular}

Data are mean \pm S.E.M normalized by the control. ${ }^{*} p<0.05$ indicates the difference in the mean values of the variable between the DLP or DLP-Lp ${ }^{\mathrm{WJL}}$ and CTL groups. ${ }^{\#} p<0.05$ indicates the difference in the mean values of the variable between the DLP-Lp ${ }^{\mathrm{WJL}}$ and DLP groups. $E^{+}=$functional endothelium present and $E-=$ absence of endothelium. Abbreviations: Phe: phenylephrine; Ach: acetylcholine; SNP: sodium nitroprusside.

and the administration of L. plantarum WJL did not correct gut microbiota dysbiosis provoked by the HFHC diet.

However, our results showed that L. plantarum WJL administration to DLP dams increased the abundance of Lachnospiraceae, a phylogenetically and morphologically heterogeneous taxon belonging to the Clostridia cluster within the Firmicutes phylum. In addition, among more than 58 genera belonging to the Lachnospiraceae family, ${ }^{33}$ we showed that $L$. plantarum WJL treatment increased the relative abundance of Blautia, Coprococcus, Dorea and Ruminococcus genera in DLP dams.

Although different genera and species of the Lachnospiraceae family are increased in cardiometabolic diseases, recent evidence shows that Lachnospiraceae might have beneficial effects on host health. ${ }^{33}$ In part, the health functions attributed to the Lachnospiraceae family may be due to their ability to produce bacterial metabolites, so called shortchain fatty acids (SCFAs). Other microbes, such as Bacteroides, Lactobacilli, Clostridium, Prevotella, and Akkermansia genera, have also been described as important SCFAs producers, mainly acetate, propionate and butyrate. ${ }^{33,34}$

An early study showed that a human-origin probiotic cocktail increases SCFA production via the augmentation of SCFA producing bacteria belonging to the Lachnospiraceae family, and Clostridium, Bacteroides, Prevotella, Oscillospira, and Akkermansia (Akkermansia muciniphila) genera in mouse and human fecal microbiota. ${ }^{35}$ In addition, it was shown that butyrate supplementation increased the Lachnospiraceae family and protected rats against high-fat diet-induced atherosclerosis, ${ }^{36}$ demonstrating an important role of butyrate in protecting from cardiovascular disease. In this way, it has been demonstrated that SCFAs, mainly acetate and butyrate, exert several metabolic effects such as the increase of fat oxidation, the reduction of body weight and dyslipidemia and the improvement of insulin sensitivity. ${ }^{36,37}$ In this way, further studies will be necessary to analyze whether the higher relative abundance of Lachnospiraceae observed in DLP dams receiving $L$. plantarum WJL contributes to an increase in SCFA production.

Previous studies demonstrated that pups from dyslipidaemic dams had low body weight at birth and weaning ${ }^{3,4}$ and the treatment of dams during the pregnancy and lactation periods with $L$. plantarum Lp62 strain triggered an increased nutritional content of milk, which may have contributed to the higher body weight observed in the pups. ${ }^{38}$ In the present study, our results did not confirm that administration of L. plantarum WJL in dams would increase body weight in offspring, demonstrating that biological properties of probiotics may be strain-specific and the success or failure of one strain under a specific physiopathological condition should not be extrapolated to another strain. Although the numeric values for the BMI and Lee index found for the rats in the present study were similar to an earlier study, ${ }^{18}$ we observed that the male offspring of DLP dams that received $L$. plantarum WJL had lower weight and length during the development, but the BMI and Lee index, important indexes to estimate body fat and obesity, were similar among the groups at 90 days of age.

Rapid weight gain in infancy, or catch-up growth, has been described as a risk factor for the development of obesity and arterial hypertension in later life. ${ }^{39,40}$ In our study, lower body weight but similar BMI and Lee index might suggest an adequate growth rate development in male offspring of DLP dams receiving $L$. plantarum $\mathrm{WJL}$, which may have impacted the cardiovascular function of those rats positively.

The etiology of arterial hypertension in rats from dams fed a high-fat diet during pregnancy and lactation includes complex mechanisms involved in central and peripheral blood pressure control. ${ }^{3,41}$ Here, we demonstrated that male offspring of dyslipidaemic dams showed increased blood pressure and endothelial dysfunction. Alterations in gut microbiota also have been found to be an important factor involved in the development of arterial hypertension programmed by maternal high-fat diet consumption, ${ }^{42}$ thus, gut microbiota-targeted interventions may represent novel effective therapeutic strategies to protect against programmed arterial hypertension.

Recently, a study showed that feeding dams a high-fat diet supplemented either with a prebiotic (long-chain inulin) or a probiotic (L. casei) during pregnancy and lactation improved maternal gut microbiota diversity and protected male offspring against hypertension..$^{10}$ In our study, L. plantarum WJL administration in maternal dyslipidemia besides being effective in protecting male offspring against arterial hypertension, was also able to restore vasorelaxation and vasoconstrictor properties in mesenteric rings. Although the underlying 
mechanisms by which the maternal administration of L. plantarum WJL prevented hypertension and endothelial dysfunction have not been explored in the present study, our results expand the scientific knowledge about its positive effects when administered during pregnancy and lactation in a metabolic dysfunctional state, such as dyslipidemia. However, previous studies have demonstrated that high SCFA production can stimulate the host G-protein-coupled receptor and olfactory receptor, such as GPR41 and Olfr78, in the smooth muscle cells of small resistance blood vessels, promoting vasodilation and lowering blood pressure. ${ }^{43}$ In this way, we highlight that further study will be needed to know if the improvement on blood pressure and endothelium dependent relaxation in DLP offspring from dams receiving L. plantarum $\mathrm{WJL}$ during pregnancy and lactation is due to high SCFA production by Lachnospiraceae.

\section{Potential limitations}

This is a pre-clinical study performed with a small sample size of dams. The lack of gut microbiota composition of the dams before the diet and treatment could be considered as a bias for the results. Additionally, the lack of female offspring could also be considered as a potential limitation of the study. The effects of maternal administration of $L$. plantarum WJL on female offspring could present more consistent results about the effects of maternal probiotic therapy on the cardiovascular function in offspring.

Additionally, the effects of maternal administration of L. plantarum WJL on offspring were tested only in male offspring.

\section{Conclusion}

In summary, our findings revealed that L. plantarum WJL administration might be a safe and promising strategy to improve lipid profile, insulin sensitivity and gut microbiota diversity in dams, and importantly to protect male offspring against programmed cardiovascular dysfunction.

\section{Abbreviations}

$\begin{array}{ll}\text { AH } & \text { Arterial hypertension } \\ \text { AP } & \text { Arterial pressure } \\ \text { AUC } & \text { Area under the curve } \\ \text { BP } & \text { Blood pressure } \\ \text { CTL } & \text { Control diet } \\ \text { TC } & \text { Total cholesterol } \\ \text { DAP } & \text { Diastolic arterial pressure } \\ \text { HR } & \text { Heart rate } \\ \text { HFHC } & \text { High-fat and high-cholesterol } \\ \text { DLP } & \text { High-fat and high-cholesterol-induced dyslipidemia } \\ \text { HDL } & \text { High-density lipoprotein } \\ \text { ITT } & \text { Insulin tolerance test } \\ \text { L. } & \text { Lactobacillus }\end{array}$

Lp Lactobacillus plantarum

LDL Low-density lipoprotein

MAP Mean arterial pressure

OTU Number of species

OGTT Oral glucose tolerance test

OD Optical density

MRS Mann, Rogosa and Sharpe

SAP Systolic arterial pressure

\section{Author contributions}

J. L. de Brito Alves, M. Magnani and H. Vidal designed the experiments. F. Leulier and K. Makki provided the Lactiplantibacillus plantarum strain. K. S. L. Guimarães and J. L. de Brito Alves performed the physiological experiments. E. Meugnier performed the cecal DNA extraction and PCR quantification of Lactiplantibacillus plantarum WJL. K. S. L. Guimarães, S. R. Noronha and J. L. de Brito Alves analyzed the data. K. S. L. Guimarães and J. L. de Brito Alves wrote the manuscript. H. Vidal, F. Leulier, S. R. Noronha, L. R. Brandão, D. A. Chianca Junior, E. Meugnier, K. Makki, V. A. Braga and M. Magnani critically reviewed the manuscript.

\section{Financial support}

This work was supported by grants from the Paraiba State Research Foundation (PRONEX, ID: 007/2019 FAPESQ-PB-MCTI/CNPq). The funding sources were not involved in the study design; in the collection, analysis and interpretation of data; in the writing of the report; or in the decision to submit the article for publication.

\section{Conflicts of interest}

The authors declare no conflict of interest.

\section{References}

1 J. J. Christensen, K. Retterstol, K. Godang, M. C. Roland, E. Qvigstad, J. Bollerslev, T. Ueland, T. Henriksen and K. B. Holven, LDL cholesterol in early pregnancy and offspring cardiovascular disease risk factors, J. Clin. Lipidol., 2016, 10, 1369-1378.

2 M. M. Mendelson, A. Lyass, C. J. O'Donnell, R. B. D'Agostino Sr. and D. Levy, Association of Maternal Prepregnancy Dyslipidemia With Adult Offspring Dyslipidemia in Excess of Anthropometric, Lifestyle, and Genetic Factors in the Framingham Heart Study, JAMA Cardiol., 2016, 1, 26-35.

3 K. S. L. Guimaraes, E. V. de Araujo, J. S. Aquino, D. A. Gadelha, C. M. Balarini, J. H. Costa-Silva, M. Magnani, H. Vidal, V. A. Braga and J. L. de Brito Alves, Effect of maternal dyslipidaemia on the cardiorespiratory 
physiology and biochemical parameters in male rat offspring, Br. J. Nutr., 2017, 118, 930-941.

4 E. V. de Araujo, L. A. Carneiro Dos Santos, G. F. F. Speretta, G. A. H. Ferreira, M. O. de Luna Freire, D. F. de Santana, A. Carvalho-Galvao, J. C. Cruz, J. H. D. Costa-Silva, V. de Andrade Braga and J. L. de Brito Alves, Short- and longterm effects of maternal dyslipidaemia on blood pressure and baroreflex sensitivity in male rat offspring, Clin. Exp. Pharmacol. Physiol., 2020, 47(1), 27-37.

5 L. Fan, S. R. Lindsley, S. M. Comstock, D. L. Takahashi, A. E. Evans, G. W. He, K. L. Thornburg and K. L. Grove, Maternal high-fat diet impacts endothelial function in nonhuman primate offspring, Int. J. Obes., 2013, 37, 254-262.

6 C. Gray, M. H. Vickers, S. A. Segovia, X. D. Zhang and C. M. Reynolds, A maternal high fat diet programmes endothelial function and cardiovascular status in adult male offspring independent of body weight, which is reversed by maternal conjugated linoleic acid (CLA) supplementation, PLoS One, 2015, 10, e0115994.

7 J. G. Robinson and N. J. Stone, The 2013 ACC/AHA guideline on the treatment of blood cholesterol to reduce atherosclerotic cardiovascular disease risk: a new paradigm supported by more evidence, Eur. Heart J., 2015, 36, 21102118.

8 R. Xie, Y. Sun, J. Wu, S. Huang, G. Jin, Z. Guo, Y. Zhang, T. Liu, X. Liu, X. Cao, B. Wang and H. Cao, Maternal High Fat Diet Alters Gut Microbiota of Offspring and Exacerbates DSS-Induced Colitis in Adulthood, Front. Immunol., 2018, 9, 2608.

9 J. L. de Brito Alves, Y. de Oliveira, N. N. C. Carvalho, R. G. S. Cavalcante, M. M. Pereira Lira, L. Nascimento, M. Magnani, H. Vidal, V. A. Braga and E. L. de Souza, Gut microbiota and probiotic intervention as a promising therapeutic for pregnant women with cardiometabolic disorders: Present and future directions, Pharmacol. Res., 2019, 145, 104252.

10 C. N. Hsu, C. Y. Hou, J. Y. H. Chan, C. T. Lee and Y. L. Tain, Hypertension Programmed by Perinatal High-Fat Diet: Effect of Maternal Gut Microbiota-Targeted Therapy, Nutrients, 2019, 11, 2908.

11 R. O. Pinheiro, P. P. Lins, J. L. P. de Carvalho, E. V. de Araujo, A. F. Alves, R. de Alencar Pereira, L. T. Toscano, A. S. Silva, E. L. de Souza, J. L. de Brito Alves and J. de Souza Aquino, Maternal dyslipidaemic diet induces sexspecific alterations in intestinal function and lipid metabolism in rat offspring, Br. J. Nutr., 2019, 1-32, DOI: 10.1017/S0007114519000011.

12 M. M. Han, J. F. Sun, X. H. Su, Y. F. Peng, H. Goyal, C. H. Wu, X. Y. Zhu and L. Li, Probiotics improve glucose and lipid metabolism in pregnant women: a meta-analysis, Ann. Transl. Med., 2019, 7, 99.

13 A. F. G. Cicero, F. Fogacci and A. Colletti, Food and plant bioactives for reducing cardiometabolic disease risk: an evidence based approach, Food Funct., 2017, 8, 2076-2088.

14 J. Zheng, S. Wittouck, E. Salvetti, C. Franz, H. M. B. Harris, P. Mattarelli, P. W. O’Toole, B. Pot, P. Vandamme, J. Walter,
K. Watanabe, S. Wuyts, G. E. Felis, M. G. Ganzle and S. Lebeer, A taxonomic note on the genus Lactobacillus: Description of 23 novel genera, emended description of the genus Lactobacillus Beijerinck 1901, and union of Lactobacillaceae and Leuconostocaceae, Int. J. Syst. Evol. Microbiol., 2020, 70(4), 2782-2858.

15 M. Schwarzer, K. Makki, G. Storelli, I. Machuca-Gayet, D. Srutkova, P. Hermanova, M. E. Martino, S. Balmand, T. Hudcovic, A. Heddi, J. Rieusset, H. Kozakova, H. Vidal and F. Leulier, Lactobacillus plantarum strain maintains growth of infant mice during chronic undernutrition, Science, 2016, 351, 854-857.

16 E. K. Kim, Y. M. Park, O. Y. Lee and W. J. Lee, Draft Genome Sequence of Lactobacillus plantarum Strain WJL, a Drosophila Gut Symbiont, Genome Announc., 2013, 1(6), e00937-13.

17 M. E. Martino, J. R. Bayjanov, P. Joncour, S. Hughes, B. Gillet, M. Kleerebezem, R. Siezen, S. A. van Hijum and F. Leulier, Resequencing of the Lactobacillus plantarum Strain WJL Genome, Genome Announc., 2015, 3(6), e0138215.

18 E. L. Novelli, Y. S. Diniz, C. M. Galhardi, G. M. Ebaid, H. G. Rodrigues, F. Mani, A. A. Fernandes, A. C. Cicogna and J. L. Novelli Filho, Anthropometrical parameters and markers of obesity in rats, Lab. Anim., 2007, 41, 111-119.

19 J. G. Caporaso, K. Bittinger, F. D. Bushman, T. Z. DeSantis, G. L. Andersen and R. Knight, PyNAST: a flexible tool for aligning sequences to a template alignment, Bioinformatics, 2010, 26, 266-267.

20 C. Lozupone and R. Knight, UniFrac: a new phylogenetic method for comparing microbial communities, Appl. Environ. Microbiol., 2005, 71, 8228-8235.

21 Z. Zhuge, L. L. Paulo, A. Jahandideh, M. C. R. Brandao, P. F. Athayde-Filho, J. O. Lundberg, V. A. Braga, M. Carlstrom and M. F. Montenegro, Synthesis and characterization of a novel organic nitrate NDHP: Role of xanthine oxidoreductase-mediated nitric oxide formation, Redox Biol., 2017, 13, 163-169.

22 C. N. Spracklen, C. J. Smith, A. F. Saftlas, J. G. Robinson and K. K. Ryckman, Maternal hyperlipidemia and the risk of preeclampsia: a meta-analysis, Am. J. Epidemiol., 2014, 180, 346-358.

23 C. J. Smith, R. J. Baer, S. P. Oltman, P. J. Breheny, W. Bao, J. G. Robinson, J. M. Dagle, L. Liang, S. K. Feuer, C. D. Chambers, L. L. Jelliffe-Pawlowski and K. K. Ryckman, Maternal dyslipidemia and risk for preterm birth, PLoS One, 2018, 13, e0209579.

24 E. Frantz, H. S. Menezes, K. C. Lange, M. P. Abegg, C. A. Correa, L. Zangalli, J. L. Vieira and C. G. Zettler, The effect of maternal hypercholesterolemia on the placenta and fetal arteries in rabbits, Acta Cir. Bras., 2012, 27, 7-12.

25 I. Khan, V. Dekou, M. Hanson, L. Poston and P. Taylor, Predictive adaptive responses to maternal high-fat diet prevent endothelial dysfunction but not hypertension in adult rat offspring, Circulation, 2004, 110, 1097-1102. 
26 A. Jarde, A. M. Lewis-Mikhael, P. Moayyedi, J. C. Stearns, S. M. Collins, J. Beyene and S. D. McDonald, Pregnancy outcomes in women taking probiotics or prebiotics: a systematic review and meta-analysis, BMC Pregnancy Childbirth, 2018, 18, 14.

27 R. M. Pigeon, E. P. Cuesta and S. E. Gililliand, Binding of free bile acids by cells of yogurt starter culture bacteria, J. Dairy Sci., 2002, 85, 2705-2710.

28 A. Costabile, I. Buttarazzi, S. Kolida, S. Quercia, J. Baldini, J. R. Swann, P. Brigidi and G. R. Gibson, An in vivo assessment of the cholesterol-lowering efficacy of Lactobacillus plantarum ECGC 13110402 in normal to mildly hypercholesterolaemic adults, PLoS One, 2017, 12, e0187964.

29 M. Begley, C. Hill and C. G. Gahan, Bile salt hydrolase activity in probiotics, Appl. Environ. Microbiol., 2006, 72, 1729-1738.

30 C. Hill, F. Guarner, G. Reid, G. R. Gibson, D. J. Merenstein, B. Pot, L. Morelli, R. B. Canani, H. J. Flint, S. Salminen, P. C. Calder and M. E. Sanders, Expert consensus document. The International Scientific Association for Probiotics and Prebiotics consensus statement on the scope and appropriate use of the term probiotic, Nat. Rev. Gastroenterol. Hepatol., 2014, 11, 506-514.

31 M. J. Foysal, R. Fotedar, M. A. B. Siddik and A. Tay, Lactobacillus acidophilus and L. plantarum improve health status, modulate gut microbiota and innate immune response of marron (Cherax cainii), Sci. Rep., 2020, 10, 5916.

32 H. Li, F. Liu, J. Lu, J. Shi, J. Guan, F. Yan, B. Li and G. Huo, Probiotic Mixture of Lactobacillus plantarum Strains Improves Lipid Metabolism and Gut Microbiota Structure in High Fat Diet-Fed Mice, Front. Microbiol., 2020, 11, 512.

33 M. Vacca, G. Celano, F. M. Calabrese, P. Portincasa, M. Gobbetti and M. De Angelis, The Controversial Role of Human Gut Lachnospiraceae, Microorganisms, 2020, 8, 573.

34 A. Barcenilla, S. E. Pryde, J. C. Martin, S. H. Duncan, C. S. Stewart, C. Henderson and H. J. Flint, Phylogenetic relationships of butyrate-producing bacteria from the human gut, Appl. Environ. Microbiol., 2000, 66, 1654-1661.

35 R. Nagpal, S. Wang, S. Ahmadi, J. Hayes, J. Gagliano, S. Subashchandrabose, D. W. Kitzman, T. Becton, R. Read and H. Yadav, Human-origin probiotic cocktail increases short-chain fatty acid production via modulation of mice and human gut microbiome, Sci. Rep., 2018, 8, 12649.

36 Y. Du, X. Li, C. Su, M. Xi, X. Zhang, Z. Jiang, L. Wang and B. Hong, Butyrate protects against high-fat diet-induced atherosclerosis via up-regulating ABCA1 expression in apolipoprotein E-deficiency mice, Br. J. Pharmacol., 2020, 177, 1754-1772.

37 E. E. Canfora, J. W. Jocken and E. E. Blaak, Short-chain fatty acids in control of body weight and insulin sensitivity, Nat. Rev. Endocrinol., 2015, 11, 577-591.

38 G. C. Messias, A. M. N. Rocha, B. M. S. Santos, A. M. Botelho, D. C. A. Silva, E. S. Porto, M. L. Dos Anjos, R. A. Sousa, M. V. Silva, T. B. da Rocha, A. V. de Melo, M. R. T. Carneiro, N. S. Aguiar, P. P. Santos, E. P. de Souza, M. P. Cruz, L. M. Marques, R. P. Rezende, C. C. Romano, A. P. Uetanabaro, G. Vinderola and R. Yatsuda, Administration of Lactobacillus plantarum Lp62 to dam rats at the end of delivery and during lactation affects TGFbeta1 level and nutritional milk composition, and body weight of pups, Eur. J. Nutr., 2019, 58, 1137-1146.

39 M. R. Sacco, N. P. de Castro, V. L. Euclydes, J. M. Souza and P. H. Rondo, Birth weight, rapid weight gain in infancy and markers of overweight and obesity in childhood, Eur. J. Clin. Nutr., 2013, 67, 1147-1153.

40 W. Perng, S. L. Rifas-Shiman, M. S. Kramer, L. K. Haugaard, E. Oken, M. W. Gillman and M. B. Belfort, Early Weight Gain, Linear Growth, and Mid-Childhood Blood Pressure: A Prospective Study in Project Viva, Hypertension, 2016, 67, 301-308.

41 E. V. de Araujo, K. S. L. Guimaraes, M. Magnani, J. C. Cruz, H. Vidal, V. A. Braga and J. L. de Brito Alves, Maternal dyslipidemia during pregnancy and lactation increases blood pressure and disrupts cardiorespiratory and glucose hemostasis in female rat offspring, Appl. Physiol. Nutr. Metab., 2019, 44, 925-936.

42 C. N. Hsu, C. Y. Hou, C. T. Lee, J. Y. H. Chan and Y. L. Tain, The Interplay between Maternal and Post-Weaning HighFat Diet and Gut Microbiota in the Developmental Programming of Hypertension, Nutrients, 2019, 11(9), 1982.

$43 \mathrm{~J}$. Ma and $\mathrm{H}$. Li, The Role of Gut Microbiota in Atherosclerosis and Hypertension, Front. Pharmacol., 2018, 9, 1082 . 